\section{S-306 THE ROLE OF ENVIRONMENTAL ASSESSMENT IN WORKPLACE COVID-19 OUTBREAK INVESTIGATION TO UNDERSTAND SARS-COV-2 TRANSMISSION}

Yiquin Chen, 'Chris Keen, Vince Sandys, Andrew Simpson, COVID-19 UK National Core Studies Consortium. ${ }^{1}$ HSE, United Kingdom

\subsection{6/OEM-2021-EPI.432}

Introduction SARS-CoV-2 is a highly transmissible novel virus that has caused the COVID-19 pandemic. Evidence is required to support effective mitigation strategies. Existing evidence has shown that the virus can be transmitted mainly through three routes: close-range airborne (droplets and aerosols), longer range inhalation of aerosol, contact with contaminated surfaces. However, their relative importance is not well understood. It is also unclear how environmental conditions can alter the dynamics of the virus transmission.

Objectives The COVID-OUT (COVID-19 Outbreak investigation to Understand Transmission) study aims to understand SARS-CoV-2 transmission routes, transmission risk factors, and the role they play in COVID-19 outbreak in workplaces.

Methods This study is part of the UK COVID-19 National Core Study (NCS) on Transmission and the Environment. The study has a series of field studies to investigate outbreaks in a range of workplaces. Each field study has serial measurements of workers and measurements in the work environments. Environmental assessment is an essential component of the study. Its data can be combined with epidemiological and laboratory data to generate hypotheses of the causes of an outbreak and can also be used to support simulation models to characterize the relative contribution of transmission routes.

Results So far three outbreak workplaces have been investigated. Preliminary findings and lessons learnt will be presented.

Conclusion The field study data collection is led by a team of occupational hygienists from the Health and Safety Executive (HSE) who work closely with epidemiologists, public health investigators, microbiologists, environmental exposure specialists to ensure critical data are collected and findings are interpreted appropriately. Occupational hygienists are skilled in assessing physical, chemical and biological hazards in workplace settings. They are equipped with established tools and frameworks for assessing risks of various hazards which can be adapted and applied in COVID-19 outbreak investigations.

\section{S-310 CANCER INCIDENCE IN AGRICULTURAL WORKERS: AN INTERNATIONAL CONSORTIUM OF AGRICULTURAL COHORT STUDIES (AGRICOH)}

${ }^{1}$ Kayo Togawa, Maria E Leon, Pierre Lebailly, Karl-Christian Nordby, Isabelle Baldi, Ewan MacFarlane, Aesun Shin, Sue Park, Robert Greenlee, Torben Sigsgaard, Ioannis Basinas, Jonathan Hofmann, Kristina Kjaerheim, Jeroen Douwes, Rachel Denholm, Gilles Ferro. 'International Agency for Research on Cancer, France

\subsection{6/OEM-2021-EPI.433}

Introduction Agricultural work can expose workers to potentially hazardous agents including known and suspected carcinogens.

Objectives We aimed to evaluate the cancer incidence in agricultural cohorts in an international consortium, AGRICOH, relative to the respective general populations.

Methods For 24 cancer sites/types and all cancers combined, we estimated standardized incidence ratios (SIRs) and 95\% confidence intervals (CIs) in eight cohorts that were linked to respective cancer registries: France (AGRICAN: $n=128,101$ ), the United States (AHS: $n=51,165$, MESA: $n=2,177$ ), Norway (CNAP: $n=43,834$ ), Australia (2 cohorts combined, Australian Pesticide Exposed Workers and Victorian Grain Farmers: $n=13,134$ ), Republic of Korea (KMCC: $n=8,432$ ), and Denmark (SUS: $n=1,899$ ). We then combined the SIR estimates across cohorts by random-effects meta-analysis.

Results During nearly 2,800,000 person-years, 23,188 cancers were diagnosed. We observed an elevated risk for melanoma of the skin (number of cohorts included $=3$, meta-SIR $=1.18$, 95\% CI: 1.01-1.38) and multiple myeloma in women $(n=4$, meta-SIR $=1.27,95 \%$ CI: 1.04-1.54) and prostate cancer $(n=6$, meta-SIR $=1.06,95 \% \mathrm{CI}: 1.01-1.12)$ compared to the general population. For several cancer sites, including bladder, breast (female), colorectum, esophagus, larynx, lung, and pancreas and all cancers combined, the risk was lower in the agricultural cohorts than in the general population $(n=7$, sexadjusted meta-SIR for all cancers combined $=0.83,95 \% \mathrm{CI}$ : $0.77-0.90)$. The direction of risk was mostly consistent across cohorts while for some cancer sites, such as liver and lung in men and women, and stomach, colorectum, and skin in men, the SIR varied greatly across cohorts.

Conclusion Our findings suggest that agricultural workers have a lower risk of various cancers and an elevated risk for prostate cancer, multiple myeloma (female), and melanoma of skin (female) compared to the general population. The observed excesses and deficits of cancer incidence in agricultural workers may be largely due to underlying differences in risk factors and warrant further investigation of specific agricultural exposures.

\section{S-318 SHIFT WORK AND THE INDIVIDUAL}

${ }^{1}$ Thomas Kantermann. ${ }^{1}$ FOM University of Applied Sciences for Economics \& Management, Germany

\subsection{6/OEM-2021-EPI.434}

Introduction Working in shifts and/or at night requires, among other things, adjusting sleep times, meal times and time for socializing. These bio-psycho-socio adaptations, when combined with wakefulness (i.e., work and exposure to environmental stimuli) during usual sleep and rest periods, have been linked to a number of negative health, wellbeing and performance outcomes. While the understanding of these negative consequences is primarily based on inductive inferences, deductive inferences for a successful identification of vulnerable or tolerant people are currently rather inconclusive.

Objectives Determination of tolerance factors for shift and night work to reduce or avoid negative consequences for health, well-being, and performance

Methods Quantitative research studies

Results One challenge to mitigate or avoid the negative consequences of shift and night work is the disparity between the individuality of shift work regulations and the inter-individual differences between employees on a biological, psychological and sociological level.

Conclusion The understanding of shift work tolerance is preliminary. The studies available on this topic differ in many aspects of study methods such as definitions of exposure and outcome variables and confounders considered. Future studies need to specify the exact shift work schedule that is 
investigated, and there needs to be a concerted effort to come to a consensus on what 'tolerance' to shift work means. Prospective studies would increase our understanding of which individual factors are associated with the development of tolerance over time, especially if they collect participants' complete occupational histories. Taking a paucity of evidence on these issues into account, the presentation will identify areas for future research with the goal of increasing evidence-based harm mitigation strategies for shift workers.

\section{S-319 NIGHT SHIFT WORK AND CANCER RISK: WHERE DO WE STAND, WHERE SHOULD WE GO?}

${ }^{1}$ Kyriaki Papantoniou. 'Medical University of Vienna, Austria

10.1136/OEM-2021-EPI.435

Introduction Night shift work was re-reclassified in 2019 by IARC/WHO as a probable human carcinogen (Group 2A) for humans, with limited epidemiological evidence for breast, prostate and colorectal cancer.

Objectives The objective of this talk is to provide an overview of the evidence on night shift work and cancer in epidemiological studies with a focus on breast cancer, to discuss strengths and limitations of existing studies and summarize areas for future research studies and policy actions.

Methods Among others, results from a pooled analysis of 5 population-based case-control studies of breast cancer using a common definition of night work (at least $3 \mathrm{~h}$ between midnight and 5 a.m.) will be presented. Results from a systematic Cochrane review on the effect of years of night shift work on cancer incidence will be summarized.

Results Women who ever worked at night had higher odds for breast cancer compared to never night workers (OR 1.12 $95 \%$ CI 1.00-1.25) in the pooled analysis. The risk was higher among pre-menopausal women (1.26; 1.06-1.51), high shift-work intensity and ER+ tumors. Our systematic review included 20 studies on breast cancer (12 case-control and 8 cohort studies). In preliminary meta-analysis, a non-linear dose-response relationship was found, with a $7 \%$ risk increase in breast cancer after 20 years of night work $(95 \%$ CI: 1.01-1.15). This finding was stronger in studies that reported lifetime occupational history and case-control studies.

Conclusions Night shift work of high intensity and long duration tends to increase the risk of breast cancer. Findings are stronger in studies with lifetime occupational history, among pre-menopausal women and positive hormone receptor subtypes. Other shift work research domains that need to be considered in future studies include 1) patterns of night work schedules 2) susceptible groups e.g. chronotype 3) critical exposure windows 4) co-exposures with occupational carcinogens.

\section{S-325 GENDERED OCCUPATIONAL INEQUALITIES AND HEALTH OVER LIFETIME: HOW CAN WE WORK THEM IN?}

${ }^{1} E$ Emilie Counil, Hyunji Byun, Mélanie Bertin. 'Institut national d'études démographiques (INED), France

10.1136/OEM-2021-EPI.436
Introduction Major economic and social changes occurred over the last 50 years, such as the increased participation of women in the labour market and the development of nonstandard forms of employment. These trends question the importance of work/employment conditions in the shaping of social inequalities in health over the life course. We thus hypothesize that (1) precarious occupational trajectories may contribute to ill health in adulthood, and that (2) they may affect genders differently.

Methods We used the French SIP (Santé Itinéraire Professionnel) national survey that collected information on occupational career and major health events of people aged 40-74 in 2006. We described poor employment conditions in terms of job instability, career discontinuity, qualification trends and versatility. We then applied multiple correspondence analysis and hierarchical ascending classification to identify patterns reflecting the accumulation of precarious employment conditions over job histories. Finally, we quantified the association between the type of occupational trajectory and self-reported health (Mini European Health Module) through multivariate logistic regression.

Results We included more than 9500 participants of workingage or $<5$-years retirees at the time of the survey. The classification showed that women were over-represented among most precarious trajectories. We also found that people with precarious trajectories more often reported less than good selfperceived health, currently experiencing longstanding illness or health problem, and activity limitation due to health problems. The trend of increasing ill health across work trajectories (stable/qualified/continuous trajectories serving as a reference) was similar among men and women, although adjusted ORs were slightly higher among women.

Conclusion We bring evidence that women experienced more precarious employment trajectories in France over the last decades than did men, with similar health outcomes among men and women. We now seek to expand our gendered perspective by taking into account hazardous working conditions as a potential mediating pathway.

\section{S-337 EXPOSURE TO PESTICIDES AND CANCER OF THE LYMPHOHEMATOPOIETIC SYSTEM IN THE AGRICOH COHORT CONSORTIUM}

${ }^{1}$ Karl-Christian Nordby, on behalf of AGRICOH Working Group on Cancer. ${ }^{1}$ National Institute of Occupational Health, STAMI, Norway

\subsection{6/OEM-2021-EPI.437}

Objective Pesticide exposure has been associated with certain cancer outcomes among farmers and applicators spraying pesticides. In the AGRICOH consortium of agricultural populations, we have evaluated cancer risk and pesticide exposure in three large cohort studies: The French Agriculture and Cancer Study - AGRICAN (FR), Agricultural Health Study (US), and Cancer in the Norwegian Agricultural Population (NO) studies.

Methods Estimates of lymphohaematologic cancers from the three cohorts (FR, US and NO) were analysed individually and then meta-analysed to yield more robust estimates of associations. Ever-never exposure to 33 chemical active ingredients from 14 chemical groups of agrochemicals was assessed using questionnaire information (US) and Crop Exposure 